\title{
Multi-wavelength, Multi-Messenger Pulsar Science in the SKA Era
}

\author{
John Antoniadis, ${ }^{1,2}$ Lucas Guillemot, ${ }^{3}$ Andrea Possenti ${ }^{*}, 4$ Slavko Bogdanov, ${ }^{5}$ \\ Josef D. Gelfand, ${ }^{6}$ Michael Kramer, ${ }^{1,7}$ Roberto Mignani, ${ }^{8,9}$ Benjamin Stappers ${ }^{7}$ \& \\ Pablo Torne ${ }^{1}$ \\ ${ }^{1}$ Max-Planck-Institut für Radioastronomie, Bonn, Germany; ${ }^{2}$ Dunlap Institute for Astronomy \\ and Astrophysics University of Toronto; ${ }^{3}$ Laboratoire de Physique et Chimie de \\ l'Environnement, Orléans, France Station de radioastronomie de Nançay, Obs. de Paris, \\ CNRS/INSU, Nançay, France; ${ }^{4}$ INAF-Osservatorio Astronomico di Cagliari, Selargius (CA), \\ Italy; ${ }^{5}$ Columbia Astrophysics Laboratory, Columbia University, New York, USA; ${ }^{6}$ New York \\ University, Abu Dhabi; ${ }^{7}$ Jodrell Bank Center for Astrophysics, University of Manchester, UK; ${ }^{8}$ \\ INAF - Istituto di Astrofisica Spaziale e Fisica Cosmica Milano, Milano, Italy; ${ }^{9}$ Kepler Institute \\ of Astronomy, University of Zielona Góra, Zielona Góra, Poland \\ email: antoniadis@dunlap.utoronto.ca
}

The Square Kilometre Array (SKA) is an integral part of the next-generation observatories that will survey the Universe across the electromagnetic spectrum, and beyond, revolutionizing our view of fundamental physics, astrophysics and cosmology. Owing to their extreme nature and clock-like properties, pulsars discovered and monitored by SKA will enable a broad range of scientific endeavour and play a key role in this quest. This chapter summarizes the pulsar-related science goals that will be reached with coordinated efforts among SKA and other next-generation astronomical facilities.

Advancing Astrophysics with the Square Kilometre Array

June 8-13, 2014

Giardini Naxos, Italy

\footnotetext{
${ }^{*}$ Speaker.
} 


\section{Introduction}

One of the key science goals for the SKA is to provide a nearly complete census of radio pulsars in the Milky Way and its Globular Clusters (Keane et al. 2015; Hessels et al. 2015). The large number of pulsar discoveries and the unprecedented timing precision of the instrument will enable a broad spectrum of science, ranging from characterization of stochastic gravitational wave (GW) signals (Janssen et al. 2015) to probing all possible outcomes of massive-star evolution (Tauris et al. 2015).

As the SKA will be detecting its first light, a fleet of sensitive telescopes will be gathering photons at all wavelengths and second generation GW detectors will be making sensitive observations. This multi-wavelength, multi-messenger frontier in astronomy will allow to tackle many remaining open problems in neutron star (NS) physics, and study with unprecedented detail the Galactic structure and content, the nature of the strong interaction, strong-field gravity and the large-scale structure of the Universe.

Figure 1 sketches an approximate timeline (design-construction-operations) for observatories that will be of particular importance for pulsar science. At radio and sub-mm wavelengths, ALMA ${ }^{1}$ and $\mathrm{EHT}^{2} / \mathrm{BlackHoleCam}^{3}$, will study the pulsar emission mechanism and magnetic structure and, jointly with pulsar-timing observations, probe the nature of strong-field gravity around the supermassive black hole (SMBH) at the center of the Milky Way.

Ground-based and space-borne instruments such as GAIA ${ }^{4}, \mathrm{LSST}^{5}, \mathrm{JWST}^{6}$ and E-ELT ${ }^{7}$ will provide a thorough census of the Galaxy's stellar content, including the companions of pulsars discovered and monitored with the SKA. Similarly, studies of NS X-ray binaries with next-generation X-ray telescopes such as NICER ${ }^{8}$, eROSITA ${ }^{9}$ and LOFT $^{10}$ and precise timing of binary millisecond pulsars will constrain the super-dense matter equation-of-state (EoS) (Watts et al. 2015) and further enrich the ensemble of laboratories for strong-field gravity (Shao et al. 2015). In $\gamma$-rays, pulsar searches in Fermi ${ }^{11}$ and $\mathrm{CTA}^{12}$ unidentified sources will allow a better understanding of the NS environment, pulsar emission and binary evolution. Beyond the electromagnetic spectrum, Advanced-LIGO ${ }^{13}$, VIRGO $^{14}$, eLISA ${ }^{15}$ and the Pulsar Timing Array (PTA) monitored by the SKA will open a new window to the GW Universe.

\footnotetext{
${ }^{1}$ Atacama Large Millimeter/submillimeter Array, http://www.almaobservatory.org/

${ }^{2}$ Event-Horizon Telescope, http://www.eventhorizontelescope.org/

${ }^{3}$ http://www.space.com/24002-black-hole-image-event-horizon.html

${ }^{4}$ Global Astrometric Interferometer for Astrophysics, http://sci.esa.int/gaia/

${ }^{5}$ Large Synoptic Survey Telescope, http://www.lsst.org/lsst/

${ }^{6}$ James Webb Space Telescope, http://www.jwst.nasa.gov/

${ }^{7}$ The European Extremely Large Telescope, https://www.eso.org/public/teles-instr/e-elt/

${ }^{8}$ Neutron star Interior Composition Explorer; http://heasarc.gsfc.nasa.gov/docs/nicer/

${ }^{9}$ extended ROentgen Survey with an Imaging Telescope Array, http://www.mpe.mpg.de/eROSITA

${ }^{10}$ Large Observatory For X-ray Timing; http://sci.esa.int/loft

${ }^{11} \mathrm{http} / / /$ fermi.gsfc.nasa.gov

${ }^{12}$ Cherenkov Telescope Array, https://www.cta-observatory.org

${ }^{13} \mathrm{https}: / / \mathrm{www}$. advancedligo.mit.edu

${ }^{14} \mathrm{http}: / /$ www.ego- gw.it/public/about/whatis.aspx

${ }^{15} \mathrm{https} / / / \mathrm{www}$.elisascience.org
} 


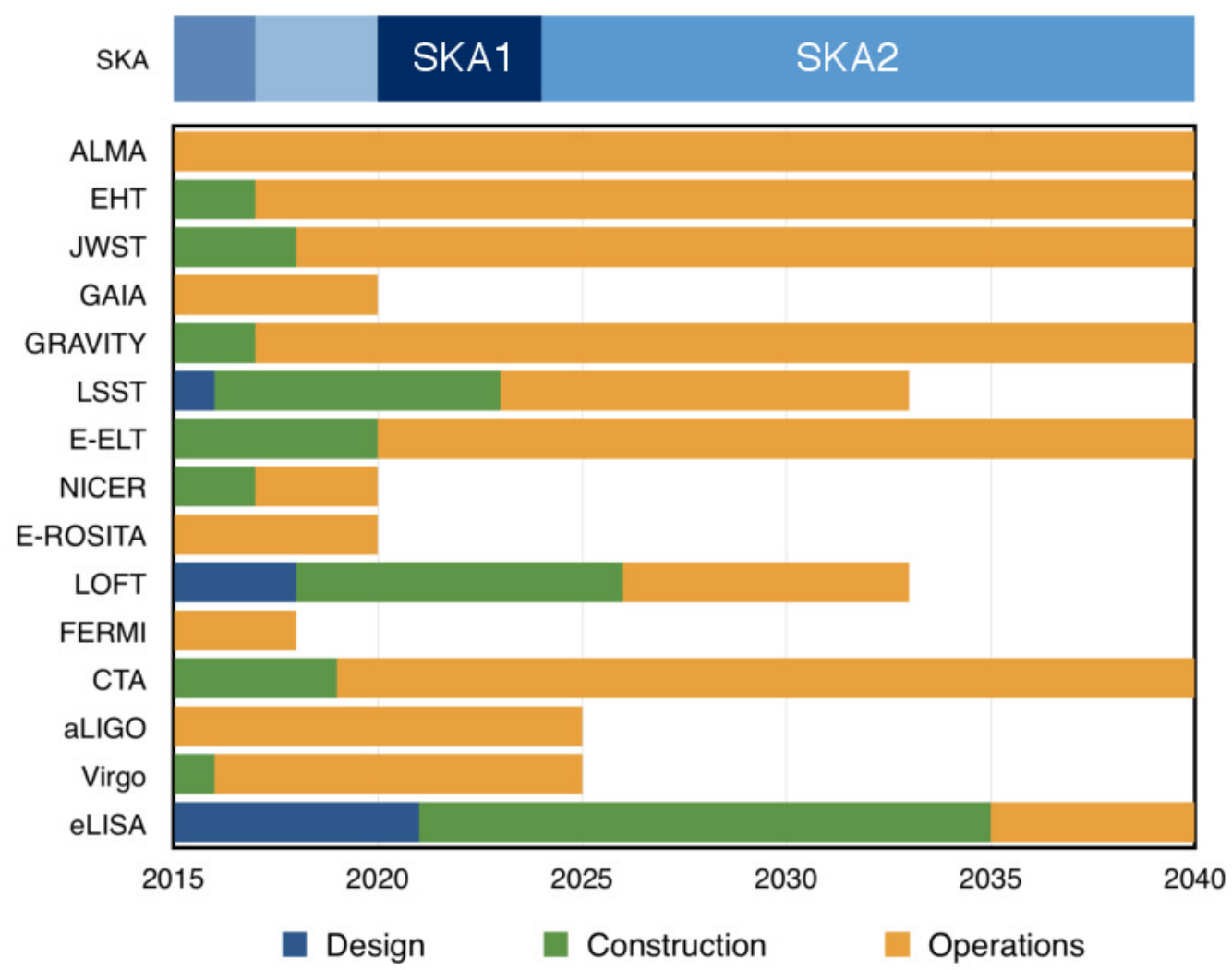

Figure 1: Approximate timeline for observatories of interest for synergistic neutron star science with the SKA.

In this chapter we elaborate on a selected number of topics, for which coordination between different observatories will provide the greatest benefits. The text is organized as follows: Section 2 covers the Galactic structure and content, focusing on studies of the Milky Way's kinematics and multi-wavelength pulsar surveys. Section 3 discusses the added benefits for stellar evolution and NS population studies. In section 4 we elaborate on the NS EoS and the nature of the Strong Interaction and in section 5 on NS-related transient phenomena. Finally, section 6 covers the multimessenger efforts in the GW detection era and section 7 concludes with some final remarks. Given space limitations, this chapter is not meant to be an in-depth review of each topic nor does it exhaust the complete potential of the multi-wavelength approach. For details on specific topics the reader is encouraged to skim through the other chapters of this book cited throughout the text.

\section{The Galactic Structure and Content}

\subsection{Targeted multi-wavelength searches for pulsars}

One of the great achievements of Fermi was the discovery by its main instrument, the Large Area Telescope (LAT), of a large number of $\gamma$-ray sources with no previously known counterparts, the so-called unassociated sources. The 2FGL catalogue (Nolan et al. 2012), a catalogue of Fermi LAT sources based on two years of data, contained 1873 sources in total, of which about $30 \%$ 
have not yet been associated with a known class of gamma-ray source; the recently published 3FGL catalogue, which is based on four years of LAT data, contains a total number of sources close to 3000. Many of these could be $\gamma$-ray pulsars, the most numerous class of Galactic $\gamma$-ray sources. Indeed, searches for pulsations at the locations of LAT unassociated sources with pulsarlike $\gamma$-ray emission properties have led to the discovery of many new pulsars, either by directly blind searching the photon data (Pletsch et al. 2012), or by conducting deep radio observations (Ray et al. 2012).

Radio searches of pulsations in LAT sources have led to the discovery of a very large number of previously unknown MSPs (Ray et al. 2012) - currently about $25 \%$ of all known in the Galaxy ${ }^{16}$. A good fraction of these new pulsars would probably have eventually been found in standard radiopulsar surveys. However, the LAT accelerated their discovery by showing radio telescopes where to look. Until the end of its mission, Fermi will continue to discover new sources and the SKA will be extremely useful in the quest for the identification of the unassociated ones. Similarly, other instruments covering different wavelengths that have started operating (e.g., GAIA or ALMA) or will start in the future (e.g., LSST, eROSITA, or the CTA), will find new sources across the spectrum that could be searched for pulsars with the SKA. To quote some examples: faint, variable optical stars detected by GAIA could point to white dwarfs orbiting unknown radio pulsars (e.g. Antoniadis et al. 2013), or "black widow" systems, with millisecond pulsars ablating their companion stars with their strong particle winds, generating optical emission modulated at the orbital period (e.g. Romani 2012). Another example is the possibility to search for young radio-emitting pulsars with the SKA, at the locations of supernova remnants, pulsar wind nebulae (PWNe) or unassociated sources discovered by CTA, in its future surveys of the very high energy $\gamma$-ray sky (Dubus et al. 2013).

\subsection{Probing the Dynamics and Structure of the Galaxy}

Regardless of the discovery method, measured positions, distances and proper motions of radio pulsars, have allowed to broadly outline the structure of the Galactic disk and, among others, unveiled the presence of a warp (Yusifov 2004). However, to date, out of $\sim 2200$ known radio pulsars, only $\sim 150$ (i.e. about $7 \%$ ) have measured proper motions and even less have measured parallaxes, fundamental in determining their actual location in the Galaxy.

The large number of pulsar discoveries expected by the SKA, both in Phase-1 (SKA1) and later in Phase-2 (Keane et al. 2015; Hessels et al. 2015), and the manifold increase in sensitivity, will improve the accuracy of previous results and allow to look for new features like humps or depletions in the Galactic disk that might have escaped previous searches. Furthermore, binary pulsars with optical counterparts, where both the proper motion and systemic radial velocity can be measured, will provide their full three- dimensional motion in the Galaxy (Lazaridis et al. 2009) enabling the reconstruction of the Milky-Way's structure and possibly unveiling thin and thick disk pulsar populations. Similarly, precise knowledge of pulsar proper motions (Smits et al. 2011) will allow to discriminate between pulsars born in the warped regions of the Galaxy from those born in the Galactic plane. In all these respects, the SKA will complement the work done by GAIA which will sample different stellar populations.

\footnotetext{
${ }^{16}$ See http://astro.phys.wvu.edu/GalacticMSPs/GalacticMSPs.txt for an up-to-date list of known Galactic disk MSPs.
} 


\subsection{The Interstellar and Intergalactic Medium}

In addition to the above, effects like dispersion measure, Faraday rotation, scattering and scintillation, routinely measured in pulsar observations, provide valuable information about the interstellar medium (ISM). Since pulsars are typically very faint, until now, mostly those located in the vicinity of the Sun have been used for studies of the ISM (Cordes \& Lazio 2002). The superb sensitivity of the full SKA will extend precision ISM studies to greater distances (Han et al. 2015) and further enable studies of the intergalactic medium (IGM) through the detection of pulsars and fast radio bursts (FRBs) in other galaxies.

If the distance to the host galaxy is known by e.g. optical and near-infrared studies of standard candles and Cepheid variables, the electron column density and magnetic field parallel to the lineof-sight to the pulsar can be calculated. These distances to nearby galaxies have been historically difficult to measure with precision (Jacoby et al. 1992), but the new and more powerful optical and infrared telescopes such as E-ELT and JWST will significantly improve distance measurements.

\section{Extreme Astrophysics and Stellar Evolution}

\subsection{Studies of the pulsar emission mechanism across the electromagnetic spectrum}

While the general concept of pulsar electromagnetic emission is fairly well established, the complex details of the radiation processes such as the exact emission heights and the relevant importance of various emission mechanisms are still unclear. Different approaches and models attempting to explain the geometry and physical processes responsible for pulsar emission rely on a broad range of assumptions that lead to different predictions (Lyne \& Graham-Smith 2012). The different techniques utilized to study the physical processes in emission regions, e.g., profile shape studies and polarimetry, make use of information at multiple frequencies across the radio spectrum (Lyne \& Graham-Smith 2012). As the radiation from pulsars is broadband, and produced presumably by several distinct radiative mechanisms, the coverage of the multiple wavelengths across the electromagnetic spectrum is required to build a complete analysis of the problem. With the SKA and the new ground and space borne observatories covering virtually the entire electromagnetic spectrum with unprecedented sensitivity and time resolution, a much better understanding of the emission processes from pulsars is possible. This new era of cutting-edge instrumentation may answer many of the related questions, that last now more than 45 years.

Beyond that, population analyses of $\gamma$-ray pulsars will yield information on the fraction of "Geminga-like" pulsars: pulsars that are only visible from high- energy observations or with extremely low radio luminosities, presumably because the radio emission beams do not cross or only graze our line of sight. The ratio of radio-loud to radio-quiet pulsars is a key observable of high-energy emission models (Watters \& Romani 2011) and the SKA's great sensitivity will be particularly useful for constraining this ratio, and thus understanding pulsar emission across the spectrum.

\subsection{Pulsar Wind Nebulae}

There will also be significant synergies between the SKA and upcoming high-energy facilities like the Cerenkov Telescope Array (CTA) regarding the study of PWNe. Powered by the rota- 
tional energy of their central NS, these objects are detected across the electromagnetic spectrum, and currently dominate the Galactic population of $\mathrm{TeV} \gamma$-ray sources (e.g. Hartman et al. 1999). The radio emission from PWNe is believed to be synchrotron emission from the electrons and positrons created in the pulsar magnetosphere interacting with the PWN's magnetic field, while its $\gamma$-ray emission is believed to result from these high-energy lepton inverse Compton scattering of background photons. Detecting both the radio and $\gamma$-ray emission from a PWN allows one to measure the electron particle spectrum, magnetic field strength, and energy density of the background photon field - critical for understanding the generation and acceleration of leptons in these objects (Gaensler \& Slane 2006; Gelfand et al. 2009). However, such an analysis is currently possible for only few sources, since many TeV PWNe remain undetected at radio wavelengths. This is likely the result of a low magnetic field strength and large angular size, resulting in a radio surface brightness too low to be detected with current facilities. However, the significant improvement in sensitivity of the SKA, especially on large angular scales, will allow us to detect radio emission from existing TeV PWNe as well as any new PWN candidate detected by CTA. Additionally, the SKA will also have the sensitivity to discover PWN and pulsars in currently unidentified TeV sources (Gelfand et al. 2015). Together, the CTA and SKA have the potential for revolutionizing our understanding of these sources.

\subsection{A multi-wavelength view of stellar evolution}

The advent of sensitive multi-wavelength surveys described above will uncover a diverse population of binary NSs. This rich NS ensemble will greatly increase the chances for revision, and ultimately unification of the stellar formation and evolution paradigm (Tauris et al. 2015). For example, the SKA will enable extremely precise mass measurements for a large number of pulsars in binaries, allowing a thorough statistical study of the mass-transfer mechanics and the distribution of NS masses at birth (Kiziltan et al. 2013; Özel et al. 2012). At the same time, sensitive optical instruments such as GAIA, LSST and E-ELT will measure the radial velocities, atmospheric composition, proper motions and parallaxes for several pulsar companions with optical counterparts, providing further information for the evolutionary history of the systems (e.g. Antoniadis et al. 2012). Furthermore, as described above, a joint optical/radio effort might increase the chances for finding unique systems. Just to give an example, intermediate-mass binary pulsars may be ideal places to look for faint, ultra-cool white dwarfs, which constraint the stellar formation history of the Milky Way and might contribute to its "dark" baryonic content (Kaplan et al. 2014). Similarly, $\mathrm{X}$-ray observatories such as LOFT may help to identify more low-mass X-ray binary/MSP transition objects which would help to understand the details of pulsar recycling models (Archibald et al. 2009; Patruno et al. 2014; Tauris et al. 2015). Deep radio observations of pulsars discovered in $\mathrm{X}$-ray or $\gamma$-ray blind searches are also key for understanding the NS luminosity distribution. Unlike high- energy signals, radio waves are dispersed by free electrons in the propagation path. The dispersion measure of pulsars inferred with radio observations yields their (approximate) distance (Cordes \& Lazio 2002), which is impossible to determine from the high-energy observations alone.

\section{Nuclear Physics and the Strong Interaction}

NSs are extremely compact objects; denser than any other object in the current Universe, and 
anything that has ever been since $\sim 3 \mathrm{~ms}$ after the Big Bang (Lattimer 2012). Owing to their extreme properties, they are of fundamental importance for studying the nature of the Strong Interaction which dictates the behaviour of matter at densities reaching and exceeding the nuclear saturation density (Watts et al. 2015). The EoS describing the bulk properties of matter can theoretically be inferred from first-principle QCD calculations. Practically however, the complicated many-body interactions at play render this approach unfeasible. Over the past few decades, numerous different approximations have been developed, leading to diverse EoS predictions that span a large space of parameters (Lattimer 2012).

The EoS of cold nuclear matter, and the way it joins up with the EoS of hot matter, uniquely determine several NS observables such as the NS mass-radius relation, moment of inertia, cooling rate, maximum spin and maximum mass above which NSs collapse to black holes. For the first time, these observables will be significantly constrained in a range of NS populations with the SKA and next-generation X-ray observatories (Watts et al. 2015). The SKA will measure masses for several hundreds of binary pulsars and significantly increase the chances for finding rapidly spinning pulsars (Watts et al. 2015; Hessels et al. 2015). Furthermore it will provide, for the first time, a direct measurement of the moment-of-inertia for pulsars in relativistic double-NS systems like J0737-3039 (Watts et al. 2015; Keane et al. 2015; Shao et al. 2015). At the same time X-ray missions such as LOFT and Athena will provide simultaneous mass and radius measurements for a handful of NSs residing in X-ray binaries and potentially measure the cooling rates of nearby, thermally emitting NSs such as Cas A (Watts et al. 2015).

Multi-wavelength targeted survey approaches may also significantly speed-up the search for EoS-constraining pulsars, by telling us where to look: fast-spinning pulsars for example are energetic and most likely radiate the bulk of their spin-down energy in the form of $\gamma$-rays (Keane et al. 2015). Furthermore, there has been increasing evidence that "black-widow" and "redback" binary pulsars that have optical, X-ray and $\gamma$-ray counterparts, might host massive NSs (van Kerkwijk et al. 2011; Romani et al. 2012). Today, precise mass measurements in these systems are challenging, mostly due to sensitivity limits of radio and optical telescopes.

\section{Transient Phenomena and the Dynamic Sky}

\subsection{Synergies between the SKA and optical telescopes}

The identification of radio transients, such as Rotating Radio Transients (RRATs) and microquasars, through the interaction with the LSST and LOFT (Feroci et al. 2012; Lazio et al. 2014), or any other X-ray sky monitor to fly in the 2020s, will be one of the main science goals of the SKA (Fenderet al. 2015). Thanks to their location in the southern hemisphere, the synergies between LSST and the SKA will be crucial to elucidate the nature of thousands of transients in the restless radio and optical sky. With its continuous monitoring of 20000 square degrees of the sky and its different observing cadence, LSST will discover thousands of transient events on time scales ranging from tens of seconds to hours over 9 decades in flux (Ivezic et al. 2008). Furthermore, the LSST will be able to respond to targets-of-opportunity (ToOs) from other facilities with a reaction time of 60s in its Rapid Response Mode. Due to its large field of view of almost 10 square degrees, the LSST will provide colour information in six bands for several fast transients at each time, following the light curve evolution before, during, and after the event and provide quick localisation 
for follow-ups with other facilities (Ivezic et al. 2008). Spectral information in the optical (including photometric redshifts for AGNs) will be crucial to complement the spectral coverage in the radio provided by the SKA and elucidate the nature of the transient, discriminating, e.g. a Galactic microquasar from an AGN. Inversely, an SKA trigger of a fast radio transient for LSST follow-up may be crucial for determining its nature. RRATs and other sort of bursting NSs would be probably undetectable in optical integration much longer than the length of the radio burst (typically a fraction of a second for the RRATs (Keane et al. 2011)), with the signal from a possible optical burst (assuming that it lasts as long as the radio burst) being washed out. While the non-detection of a candidate RRAT in the optical down to mag 24.5 (the typical sensitivity of $2 \times 15$ s-long LSST snapshot integration) would still be provide information to help identify its NS, more intriguing synergies would emerge with the E-ELT if it is equipped with suited instruments to fully exploit its potentials in time-domain astronomy down to the ms time scales. The detection of simultaneous optical and radio bursts from RRATs in coincidence with the radio burst, a goal that we missed so far, will shed light on the origin of these events and will allow to test the proposed models by, e.g. comparing the optical and radio fluence, the profile of the burst light curve, and the characteristics of the radio and optical pulsations (typically detected during a RRAT burst), including possible time lags.

\subsection{Transients with the SKA and X-ray Telescopes}

The real-time identification of Galactic X-ray transients and follow-up of variable sources discovered by LOFT, eROSITA and, a posteriori, by Fermi is another field that will greatly benefit from the synergy with the SKA. For example LOFT, with its Wide Field Monitor (WFM), which will cover at least $50 \%$ of the sky simultaneously in the $2-50 \mathrm{keV}$ energy band, will be a perfect discovery machine of X-ray transients, such as magnetars, which have been well known and extensively studied for the past three decades. However, well known does not necessarily mean well understood. After the discovery of the prototype source back in 1979, only 30 magnetars have been found (including candidates) and we are still in a position where better understanding would benefit from the discovery of more sources. The recent discovery of both radio-loud (e.g. Shannon \& Johnston 2013) and low magnetic field magnetars (Rea et al. 2010), has triggered a profound rethinking of the nature of these objects. Most magnetars have been identified by their transient Xray emission. Previous large field-of-view telescopes, such as the All-Sky Monitor aboard RXTE, were instrumental in this role, spotting several candidates. With a factor of 20 larger collecting area, the WFM will discover many more magnetar candidates, triggering alerts for other new facilities, including the SKA. Following-up magnetars in radio during, but not only, their bursting phase is key to solve the long-standing dichotomy on the magnetar radio-quietness or radio-loudness and peer deep into the very nature of magnetars.

Other types of erratic variability in radio pulsars can be explored by the LOFT and SKA. A few pulsars tend to emit Giant Radio Pulses (GRPs) and, so far, similar phenomena at different energies (Giant Optical Pulses; Strader et al. 2013) have been observed to occur simultaneously in the Crab pulsar. Do they occur in the X rays as well? Only the SKA and LOFT will be able to answer this question. Phenomena discovered only recently in radio pulsars are the mode switches observed synchronously in X rays and in radio, like e.g. in PSR B0943+10 (Hermsen et al. 2013). How many other radio pulsars show a similar behaviour? The SKA and LOFT will certainly find 
more of such cases. On longer time scales, the nature of the many variable X-ray sources that eROSITA will discover in its 4 year all-sky survey ( 8 scans in total) could be clarified by the SKA and optical facilities.

\subsection{Fast Radio Bursts}

Finally, an emerging field where synergy may be proven useful is the discovery and characterization of FRBs. FRBs are temporary isolated impulsive bursts of radio emission with very short duration of a few milliseconds. An initial discovery by Lorimer et al. (2007) was followed by a small number of detections (Keane et al. 2012; Thornton et al. 2013; Spitler et al. 2014) which dissipated the initial doubts about the astrophysical origin of the bursts. The sources of FRBs remain elusive but their large measured dispersion measures and sky locations suggest a possible extragalactic origin. If this is the case, apart from the importance of discovering a likely new population of astronomical sources at cosmological distances, they will become important tools for the study of the intergalactic medium (Ginzburg 1973). The inherent short duration of FRBs make their detection very difficult in current radio surveys, where a big single-dish telescope with its small field of view is scanning the whole sky on many-year timescales. Additionally, the commonly adopted off-line processing of survey data usually leads to detection of events well after they have occurred on the sky. What makes these phenomena even more intriguing is that no long-lasting counterpart in radio or other wavelengths is found in the direction of the detected bursts. This could mean that additional radiation of the burst at other wavelengths, if any, is also short-lived. The SKA's wide field of view will allow to monitor big portions of the sky at once, increasing significantly the detection rate for these FRBs, which are thought to occur several thousand times-per-day per-sky (Thornton et al. 2013; Spitler et al. 2014). In addition, the proposed real-time processing back-end for the survey data in search of short radio bursts enable the necessary rapid follow-up of the detected events. A real-time warning from an SKA detection could trigger rapid ToO observations at other wavelengths in the burst direction. The SKA in combination with the observatories at all other wavelengths will be key to resolve the mystery of the origin of FRBs and let us study in detail the sources and the physical processes producing these unexpected bursts of radiation.

\section{Strong-Field Gravity and the Large-Scale Structure}

\subsection{Studies of the Galactic-Centre black hole across the spectrum}

Observations of the orbits of the so-called "S-stars" have provided detailed information about the object in the centre of our Galaxy. The observations provide the most convincing case for it being a super-massive black hole (Genzel et al. 2010; Melia \& Falcke 2001). First detected in the radio as a point source named Sgr A* (Sagittarius A*), the source is now being studied also at near-infrared and X-ray wavelengths. Tracing the orbits of the S-stars, one can derive the distance to the Galactic Centre $(8 \mathrm{kpc}$ ) and the mass of the black hole (4 million solar masses). Finding (even normal) pulsars orbiting Sgr $\mathrm{A}^{*}$, the spin and the quadrupole moment can be determined with high precision (Eatough et al. 2015). These measurements can be compared with constraints to be derived with high-precision optical astrometry of the inner-most stars. Measuring for instance the mass of Sgr A* with radio pulsars to a precision of one solar mass (Eatough et al. 2015), we 
can determine the distance to the Galactic Centre using the optical observation with a precision to about $1 \mathrm{pc}$, providing a firm anchor for our understanding of the Galactic dynamics.

\subsection{Multi-Messenger Gravitational Wave Science}

Precision timing with the SKA will start an era of GW astronomy with pulsars. SKA1 will virtually guarantee the detection of a stochastic GW background that has emerged from a population of super-massive binary black holes that was present in processes of early galaxy formation (Janssen et al. 2015). Full SKA will allow studying this background in great detail, thereby, for instance, providing insight into the fundamental properties of gravitons such as their spin and mass (Lee et al. 2010). In general, the SMBH population that is detectable with a PTA experiment is of higher mass than those of sources detectable with the space- based GW detector eLISA. With PTAs being sensitive to SMBHs with $10^{7}$ solar masses and orbital periods between 10 and 20 years, SKA observations provide a truly complementary window to the SMBH population. Moreover, observations with the SKA can provide measurements of the amplitude and spectral shape of the GW background, which encodes information about galaxy merger and SMBH accretion processes. As for instance pointed out by Sesana (2013), the amplitude of the signal tracks the number of occurred mergers integrated over the redshift range, while the spectral shape should contain a break frequency where contribution of individual systems becomes important. Indeed, some individual sources may produce a signal that is significantly larger than indicated by the average spectrum, allowing the detection of a single source which will eventually evolve into the eLISA frequency band.

In a comprehensive review Burke-Spolaor (2013) discusses in more detail the possibilities, and importance, of electromagnetic identification of the sources of GWs, which could be from continuous-wave, burst or GW memory sources. PTAs are already being used to dis-prove the identification of supermassive black hole binaries in the local neighbourhood Jenet et al. (2004). As the sensitivity of PTAs improves dramatically with the SKA, improved limits and ultimately detections will be possible. Localising these sources by use of an electromagnetic counterpart such as might be possible with current surveys in optical and X-ray wavelengths and using future facilities like LSST, IXO/Athena, Astro-H combined with the GW signature will allow significantly more detailed information about the binary system to be determined.

For compact relativistic binaries with sufficiently small orbital periods, it is possible that GWs may be directly detectable with eLISA. The orbital frequency of the Double Pulsar, for instance, is $1.16 \times 10^{-4} \mathrm{~Hz}$, so that we can expect to observe a strain of about $5 \times 10^{-21}$ at $2.3 \times 10^{-4}$ $\mathrm{Hz}$ (Kramer \& Stairs 2008). Realistically, a detection may be aggravated by the large expected background of double-white-dwarf systems with similar orbital periods (Nelemans et al. 2001). However, if the orbital ephemerides are well known from radio timing with the SKA, and because the systems should also produce power at the next orbital harmonic, it should be possible to detect the appropriate sources in a coherent search that takes advantage of the known direction to the source (S. Sigurdsson \& C. Miller, private communication). If detection is made, it is possible to combine the observations obtained from the radio with those obtained with eLISA. This combination should in principle be able to provide the exact distance to the source, the true inclination angle of the system (rather than either sine or cosine of the inclination angle) and the masses. Therefore, 
the system should be vastly over-determined, allowing to provide unprecedented tests of theories of gravity.

Isolated neutron stars may also be the source of GWs if they are deformed in such a way as to make them axi-symmetric. The GW amplitude will depend on the size of the asymmetry which in turn is strongly dependent on the nature of the equation of state of the neutron star and the strength of the internal magnetic fields. Significant and important limits on the degree of deformation and the fraction of the spin-down energy loss of pulsars that might manifest as GW emission have been obtained with current generation GW observatories (e.g. Aasi et al. 2014; The LIGO Scientific Collaboration et al. 2014) however the SKA will be operating at the same time as the much more sensitive advanced LIGO and VIRGO detectors. To be able to undertake these searches for GWs typically involves long integration times and it is therefore necessary to have good models of the rotational history of the pulsars. The SKA will help by discovering many more pulsars, thus improving chances of finding even just one deformed source and also be enabling the monitoring of even larger numbers of pulsars. Moreover we will also be sensitive to systems that might exhibit GW bursts. This is only the tip of what might be possible though synergies between the groundbased gravitational wave observatories and the SKA.

\section{Conclusions}

In this chapter we elaborated on various aspects of the multi-wavelength, multi-messenger NS science that will be enabled in the SKA era. Current simulations show that even SKA1 can discover a total of about 10000 normal pulsars and perhaps as many as 1800 millisecond pulsars (MSPs), with SKA1-LOW surveying the sky with the Galactic latitude $|b| \geq 5^{\circ}$, and SKA1-MID surveying the sky with the Galactic latitude $|b| \leq 10^{\circ}$ (Keane et al. 2015). Full SKA will provide a complete census of radio pulsars and with its Aperture Array systems should allow for an optimum combination of sensitivity, field-of-view and number of beams to be able to obtain exceptional cadence on a very large number of sources. These key features will allow for significant advances in our understanding of NSs, which could be further accelerated by coordinated efforts with other next-generation telescopes. As demonstrated here, synergies across the electromagnetic-spectrum and beyond could provide a better understanding of (I) the Galactic structure and content, (II) extreme astrophysics and stellar evolution, (III) nuclear physics and the strong interaction, (IV) transient phenomena and (V) strong-field gravity and the large-scale structure of the Universe.

\section{References}

Aasi, J., Abadie, J., Abbott, B. P., et al. 2014, ApJ, 785, 119

Antoniadis, J., Freire, P. C. C., Wex, N., \& et al. 2013, Science, 340, 448

Antoniadis, J., van Kerkwijk, M. H., Koester, D., \& et al. 2012, MNRAS, 423, 3316

Archibald, A. M., Stairs, I. H., Ransom, S. M., \& et al. 2009, Science, 324, 1411

Burke-Spolaor, S. 2013, Classical and Quantum Gravity, 30, 224013

Cordes, J. M., \& Lazio, T. J. W. 2002, ArXiv Astrophysics e-prints, ArXiv:0207156

Dubus, G., Contreras, J. L., Funk, S., et al. 2013, Astroparticle Physics, 43, 317 
Eatough, R. P., Lazio, T. J. W., Casanellas, J., et al. 2015, "Observing Radio Pulsars in the Galactic Centre with the Square Kilometre Array", in proc. Advancing Astrophysics with the Square Kilometre Array, PoS(AASKA)045

Fender, R. et al. 2015, "The Transient Universe with the Square Kilometre Array", in proc. Advancing Astrophysics with the Square Kilometre Array, PoS(AASKA)051

Feroci, M., Stella, L., van der Klis, \& et al. 2012, Experimental Astronomy, 34, 415

Gaensler, B. M., \& Slane, P. O. 2006, ARA\&A, 44, 17

Gelfand, J. D., Breton, R. P., Ng, C.-Y., et al. 2015, "Pulsar Wind Nebulae in the SKA era", in proc. Advancing Astrophysics with the Square Kilometre Array, PoS(AASKA)046

Gelfand, J. D., Slane, P. O., \& Zhang, W. 2009, ApJ, 703, 2051

Genzel, R., Eisenhauer, F., \& Gillessen, S. 2010, Reviews of Modern Physics, 82, 3121

Ginzburg, V. L. 1973, Nature, 246, 415

Han, J. L., van Straten, W., Lazio, T. J. W., et al. 2015, "Three-dimensional Tomography of the Galactic and Extragalactic Magnetoionic Medium with the SKA", in proc. Advancing Astrophysics with the Square Kilometre Array, PoS(AASKA)043

Hartman, R. C., Bertsch, D. L., Bloom, \& et al. 1999, ApJS, 123, 79

Hermsen, W., Hessels, J. W. T., \& Kuiper, e. 2013, Science, 339, 436

Hessels, J. W. T., Possenti, A., Bailes, M., et al. 2015, "Pulsars in Globular Clusters with the SKA", in proc. Advancing Astrophysics with the Square Kilometre Array, PoS(AASKA)047

Ivezic, Z., Tyson, J. A., Acosta, E., et al. 2008, ArXiv e-prints, arXiv:arXiv:0805.2366

Jacoby, G. H., Branch, D., Ciardullo, R., Davies, R. L., \& et al. 1992, PASP, 104, 599

Janssen, G. H., Hobbs, G., McLaughlin, M., et al. 2015, "Gravitational Wave Astronomy with the SKA", in proc. Advancing Astrophysics with the Square Kilometre Array, PoS(AASKA)037

Jenet, F. A., Lommen, A., Larson, S. L., \& Wen, L. 2004, ApJ, 606, 799

Kaplan, D. L., Boyles, J., Dunlap, B. H., \& et al. 2014, ApJ, 789, 119

Keane, E. F., Kramer, M., Lyne, A. G., Stappers, B. W., \& McLaughlin, M. A. 2011, MNRAS, 415, 3065

Keane, E. F., Stappers, B. W., Kramer, M., \& Lyne, A. G. 2012, MNRAS, 425, L71

Keane, E. F., Bhattacharyya, B., Kramer, M., et al. 2015, "A Cosmic Census of Radio Pulsars with the SKA", in proc. Advancing Astrophysics with the Square Kilometre Array, PoS(AASKA)040

Kiziltan, B., Kottas, A., De Yoreo, M., \& Thorsett, S. E. 2013, ApJ, 778, 66

Kramer, M., \& Stairs, I. H. 2008, ARA\&A, 46, 541

Lattimer, J. M. 2012, Annual Review of Nuclear and Particle Science, 62, 485

Lazaridis, K., Wex, N., Jessner, A., \& et al. 2009, MNRAS, 400, 805

Lazio, J. W., Kimball, A., Barger, A. J., \& et al. 2014, PASP, 126, 196

Lee, K., Jenet, F. A., Price, R. H., Wex, N., \& Kramer, M. 2010, ApJ, 722, 1589

Lorimer, D. R., Bailes, M., McLaughlin, M. A., Narkevic, D. J., \& Crawford, F. 2007, Science, 318,777

Lyne, A., \& Graham-Smith, F. 2012, Pulsar Astronomy

Melia, F., \& Falcke, H. 2001, ARA\&A, 39, 309

Nelemans, G., Yungelson, L. R., \& Portegies Zwart, S. F. 2001, A\&A, 375, 890

Nolan, P. L., Abdo, A. A., Ackermann, M., et al. 2012, ApJS, 199, 31

Özel, F., Psaltis, D., Narayan, R., \& Santos Villarreal, A. 2012, ApJ, 757, 55 
Patruno, A., Archibald, A. M., Hessels, J. W. T., \& et al. 2014, ApJ, 781, L3

Pletsch, H. J., Guillemot, L., Allen, B., \& et al. 2012, ApJ, 744, 105

Ray, P. S., Abdo, A. A., Parent, D., \& et al. 2012, ArXiv e-prints, arXiv:ArXiv:1205.3089

Rea, N., Esposito, P., Turolla, R., \& et al. 2010, Science, 330, 944

Romani, R. W. 2012, ApJ, 754, L25

Romani, R. W., Filippenko, A. V., Silverman, J. M., \& et al. 2012, ApJ, 760, L36

Sesana, A. 2013, MNRAS, 433, L1

Shannon, R. M., \& Johnston, S. 2013, MNRAS, 435, L29

Shao, L., Stairs, I. H., Antoniadis, J., et al. 2015, "Testing Gravity with Pulsars in the SKA Era", in proc. Advancing Astrophysics with the Square Kilometre Array, PoS(AASKA14)042

Smits, R., Tingay, S. J., Wex, N., Kramer, M., \& Stappers, B. 2011, A\&A, 528, A108

Spitler, L. G., Cordes, J. M., Hessels, J. W. T., \& et al. 2014, ArXiv e-prints, arXiv:ArXiv:1404.2934

Strader, M. J., Johnson, M. D., Mazin, B. A., \& et al. 2013, ApJ, 779, L12

Tauris, T. M., Kaspi, V. M., Breton, R. P., et al. 2015, "Understanding the Neutron Star Population with the SKA", in proc. Advancing Astrophysics with the Square Kilometre Array, PoS(AASKA)039

The LIGO Scientific Collaboration, the Virgo Collaboration, Aasi, J., et al. 2014, ArXiv e-prints, arXiv: 1410.8310

Thornton, D., Stappers, B., Bailes, M., \& et al. 2013, Science, 341, 53

van Kerkwijk, M. H., Breton, R. P., \& Kulkarni, S. R. 2011, ApJ, 728, 95

Watters, K. P., \& Romani, R. W. 2011, ApJ, 727, 123

Watts, A., Xu, R., Espinoza, C., et al. 2015, "Probing the neutron star interior and the Equation of State of cold dense matter with the SKA", in proc. Advancing Astrophysics with the Square Kilometre Array, PoS(AASKA14)043

Yusifov, I. 2004, in The Magnetized Interstellar Medium, ed. B. Uyaniker, W. Reich, \& R. Wielebinski, 165-169 